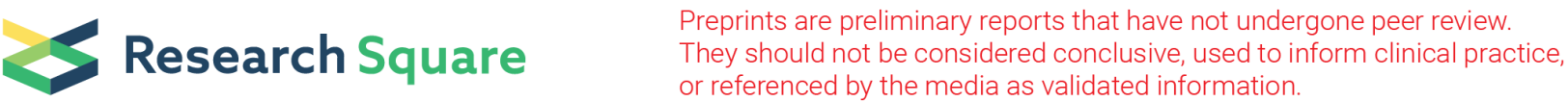

\section{A New Risk Score Model Based on Lactate Dehydrogenase Predicting Prognosis in Esophageal Squamous Cell Carcinoma Treated With Chemoradiotherapy}

Jinmin Han

Shandong cancer Hospital

Tao Zhou

Shandong Cancer Hospital

Chengxin Liu

Shandong Cancer Hospital

Baosheng Li ( $\sim$ baoshle1963@163.com )

Department of Radiation Oncology, Shandong Cancer Hospital and Institute, Shandong First Medical University and Shandong Academy of Medical Sciences, Jinan 250117, China https://orcid.org/00000002-1515-2918

\section{Research}

Keywords: pretreatment serum LDH level, personalized management, esophageal squamous cell cancer (ESCC)

Posted Date: September 8th, 2020

DOI: https://doi.org/10.21203/rs.3.rs-70861/v1

License: (c) (i) This work is licensed under a Creative Commons Attribution 4.0 International License. Read Full License 


\section{Abstract}

Purpose: The current study was to assess the prognostic value of the lactate dehydrogenase (LDH) in esophageal squamous cell cancer (ESCC) patients and to generate a risk score model to predict prognosis in patients who undergone chemoradiotherapy. Patients and Methods: 614 ESCC patients who received chemoradiotherapy were performed from 2012 to 2016. The optimal cutoff points for continuous variables were calculated by the X-tile program. We analyzed the association between LDH level and clinicopathological characteristics. And a 1:3 propensity score matching analysis was used to compensate for differences in baseline characteristics. The Kaplan-Meier methods and Cox regression models were used to explore the prognostic factors for overall survival (OS) and progression-free survival (PFS). Based on the results, we developed a corresponding risk score model and assessed its predictive capacity in the subgroups. Results: The optimal cutoff points of age, CEA, Cyfra21-1, tumor length, total dose and LDH were defined as follows: 69 years, $2.4 \mathrm{ng} / \mathrm{ml}, 6.4 \mathrm{ng} / \mathrm{ml}, 6.5 \mathrm{~cm}, 58.8 \mathrm{~Gy}$ and $134 \mathrm{U} / \mathrm{L}$, respectively. A high level of $L D H$ was associated with advanced $M$ stage $(p=0.005)$ and larger tumor length $(p=0.026)$. Patients in the high-LDH group had shorter PFS and worse OS than those in the lowLDH group. Multivariate survival analysis indicated that pretreatment serum LDH level $(p=0.039) \rrbracket$ Cyfra21-1 level $(p=0.003)$, tumor length $(p=0.013)$, clinical $N$ stage $(p=0.047)$ and clinical $M$ stage $(p=0.011)$ were independent predictors for OS. Furthermore, a risk score model based on these five prognostic factors was established to divide patients into three groups with obvious prognosis $\left(\chi^{2}=\right.$ 20.53, $p<0.0001)$. Conclusion: Pretreatment serum LDH levels may be a reliable factor in predicting the therapeutic effect of chemoradiotherapy in ESCC. A risk score model combined LDH, Cyfra21-1 and other prognostic factors could help to guide a personalized management. Further validation is needed before widely used in clinical practice.

\section{Introduction}

Esophageal cancer $(E C)$ is a frequent malignancy with an increasing incidence worldwide, ranking the fourth leading causes of cancer-related death in China. ${ }^{1,2}$ More than $85 \%$ of all EC cases were diagnosed with esophageal squamous cell carcinoma (ESCC). ${ }^{3}$ Despite advancement in therapeutic strategies have improved the prognosis of ESCC patients, the long-term survival still remains dismal. ${ }^{4}$ As we all know, TNM staging system has been correlated with survival in predicting prognosis for ESCC. However, the clinical outcomes vary greatly even at the same stage. Therefore, identifying potential indicators and establish an accurate and dependable prediction model for evaluating the prognosis of patients with ESCC before treatment is of great importance in clinical practice.

Lactate dehydrogenase (LDH) is an important enzyme involved in anaerobic glycolysis. It has been reported that $\mathrm{LDH}$ is not only involved in tumor occurrence but also plays an important role in tumor maintenance and progression. 5,6 Serum LDH, which is easily available in routine clinical practice, exists in various types of human tissue and neoplasms, and elevated LDH levels have traditionally been regarded as a marker of high tumor burden, which is a poor prognostic factor in various cancer. ${ }^{7}$ Growing evidences 
have been contributed to explore the relationship between LDH and the progression of tumorigenesis in different malignancies, such as in lymphoma, ${ }^{8}$ duodenum adenocarcinoma, ${ }^{9}$ melanoma, ${ }^{10}$ breast cancer, ${ }^{11}$ nasopharyngeal carcinoma, ${ }^{12}$ lung cancer, ${ }^{13}$ pancreatic carcinoma. ${ }^{14}$ Several investigators also scrutinized the prognostic value of LDH in ESCC. ${ }^{15-20}$ However, owing to the inconsistent conclusions, serum LDH is a controversial prognostic biomarker for prognosis in ESCC and needs to be further investigated. Furthermore, studies on the influence of LDH on the prognosis are still lacking for patients with ESCC underwent chemoradiotherapy. Thus, we aimed to investigate the prognostic value of LDH in patients with ESCC who receiving chemoradiotherapy in this work. Then we performed univariate and multivariate analyses to identify the prognostic factors in ESCC patients. Based on the results of the multivariate analysis, we designed a risk score model for exploring the prognosis of ESCC patients undergone chemoradiotherapy.

\section{Materials And Methods}

\section{Patient Characteristics and study design}

In the present study, data on 614 patients were retrieved from the database of our hospital from January 1, 2012 to December 31, 2016. All patients had either rejected surgery or were unable to operation. The Criteria for entering this study included the following: (1)pathologically or cytologically proved ESCC with clinical stage II-IVa, excluding $\mathrm{IV}_{\mathrm{b}}$;(2)receipt of chemoradiotherapy before recurrence or progression; (3)no patients with any form of acute or chronic inflammatory diseases or infections, such as acute myocardial infarction, acute hepatitis B virus infection, acute cholecystitis and bone diseases; (4)no evidence of prior malignant carcinoma during the past five years. The clinical data were collected from the medical records: gender, age, Performance Status, tumor node metastasis (TNM) stage, tumor length, baseline LDH level, date of diagnosis and recurrence date. All the pathological diagnoses were confirmed by pathologists in our department. The TNM stage in this study was in accordance with the AJCC/UICC TNM staging system (the 7th edition). ${ }^{21}$ The PS was defined according to the criteria of Eastern Clinical Oncology Group (ECOG) ${ }^{22}$ Our study also included some previously identified prognostic factors to adjust the prognostic effect of LDH, such as cytokeratin 19 fragment antigen 21-1 (Cyfra21-1), carcinoembryonic antigen (CEA). Those patients with incomplete medical records were further excluded. All registered patients have written informed consent. The study was approved by the Institutional Review Board of Shandong Cancer Hospital.

\section{Treatment protocol and follow-up}

The therapeutic strategies were based on the National Comprehensive Cancer Network (NCCN) Clinical Practice guidelines. All participants underwent three-dimensional conformal radiotherapy (3D-CRT) or intensity modulated radiotherapy (IMRT). They underwent radiotherapy for 4-7 weeks, receiving a total dose of 45-70 Gy. Each ESCC patient received concurrent chemoradiotherapy (CCRT) or sequential chemoradiotherapy (SCRT) based on individualized treatment strategy. The chemotherapy regimens mainly include cisplatin plus 5-fluorouracil or cisplatin plus paclitaxel. Survival information, including 
Overall survival (OS) and progression-free survival (PFS), was concluded until October 31, 2018. OS was defined as the date of pathological diagnosis to the date of death or last follow-up. The definition of the PFS interval was between the date of pathological diagnosis and the date of disease progression or the date of death or last contact. Follow-up was performed at regular intervals of every 3-6 months.

\section{Statistical analyses}

Statistical analyses were performed using SPSS version 24.0 (IBM Corp, NY, USA), R, version 2.15.3 (R Project for Statistical Computing) and GraphPad Prism 7.0. The continuous variables were stratified into two groups by the optimal cut-off points using the X-tile program. ${ }^{23}$ The chi-squared tests were used to compare categorical variables data between two groups. The OS and PFS were analyzed with KaplanMeier method using GraphPad Prism 7.0. A 1:3 optimal propensity score-matched method was used to control confounding. ${ }^{24}$ The Propensity Scores were estimated using a multivariable logistic regression model. Univariable and Multivariable Cox proportional hazards regression methods were used to identify independent risk factors of ESCC. Statistical significance was accepted at the $\mathrm{P} \leq 0.05$ level.

\section{Results}

\section{Patient characteristics}

614 ESCC patients were recruited for this study. There were 478 (77.9\%) males and $136(22.1 \%)$ females, with the median age was 63 years (range 35-85). According to the X-tile program, the optimal cut-off points for age, CEA, Cyfra21-1, tumor length, total dose as well as LDH were defined as follows: 69 years, $2.4 \mathrm{ng} / \mathrm{ml}, 6.4 \mathrm{ng} / \mathrm{ml}, 6.5 \mathrm{~cm}, 58.8 \mathrm{~Gy}$ and $134 \mathrm{U} / \mathrm{L}$, respectively. The X-tile analyses for LDH were shown in Figure 1.The patients then were stratified into low ang high groups based on LDH for further analyses (LDH $\leq 134$ and LDH >134), a total of $546(88.9 \%)$ patients with high-LDH were stratified into elevated group, whereas $68(11.1 \%)$ patients were classified into decreased group.

We found that patients with a high-LDH level was associated with more advanced cM stage $(P=0.005)$ and larger tumor length $(P=0.026)$. No statistically significant association was observed between LDH and other clinical features. To balance differences in the clinical features among groups, all patients were randomly selected and matched according to a 1:3 ratio from each group with similar characteristics. After matching, a total of 256 patients were matched successfully, with 68 patients in low-LDH group and 188 in high-LDH group. Patients' clinical features were balanced between the low-LDH group and the high-LDH group after matching. The correlation between patient characteristics with LDH were summarized in Table 1.

\section{Prognostic value of Pretreatment serum LDH levels}

In the whole cohort, the median PFS times were 31.5 and 17.5 months for the low-LDH group and the high-LDH group, respectively. And the median OS times were 32.4 and 25.5 months for the group and the high-LDH group, respectively. It was worthy to note that more than half of the patients in the low-LDH 
group survived at the last follow-up. Before matching, the patients in the high-LDH group had significantly shorter PFS and worse OS than those in the low-LDH group by Kaplan-Meier method (all log-rank $p<0.05$, Figure S1).

For the matched cohort, Kaplan-Meier analysis showed that the PFS and OS rates in the high-LDH group were significantly shorter than the low-LDH group, as listed in Figure 2.The survival curves on OS of Cyfra21-1 level $(p=0.0004)$, tumor lengt $(p=0.0162)$, $c N$ stage $(p=0.0098)$, $c M$ stage $(p=0.0198)$ were shown in Figures 3A-D, respectively.

\section{Survival Risk Using Univariate and Multivariate Cox Regression Analysis}

The results of univariate and multivariate Cox regression analyses of PFS and OS after chemoradiotherapy of ESCC patients before matching are listed in Table S1 and Table S2. After matching, in the univariate Cox regression analysis of PFS, Cyfra21-1 levels, LDH levels as well as tumor length were significantly associated with tumor recurrence (all $p<0.05$ ). And we also found significant correlations between the following characteristics and OS in the univariate analysis: Cyfra21-1 levels, tumor length, $\mathrm{CN}$ stage, $\mathrm{cM}$ stage and LDH levels (all $\mathrm{p}<0.05$ ). Then the significant factors were subjected to the multivariate analysis to identify independent prognostic factors. Multivariate analysis revealed that Cyfra21-1 levels (HR: 2.37; 95\% Cl: 1.22-4.60; $p=0.011$ ), LDH levels (HR: 1.50; 95\% Cl: 1.03-2.19; $p$ $=0.035)$ as well as tumor length (HR: 1.62; 95\% Cl: 1.15-2.28; $p=0.005)$ were independent factors affecting PFS in patients with ESCC, while Cyfra21-1 levels (HR: 2.81; 95\% Cl: 1.43-5.50; $p=0.003$ ), tumor length (HR: $1.61 ; 95 \% \mathrm{Cl}: 1.11-2.34 ; \mathrm{p}=0.013)$, cN stage (HR: 1.94; 95\% Cl: $1.01-3.72 ; \mathrm{p}=0.047), \mathrm{cM}$ stage (HR: $2.04 ; 95 \% \mathrm{Cl}: 1.18-3.53 ; p=0.011$ ) and LDH levels (HR: $1.56 ; 95 \% \mathrm{Cl}: 1.02-2.39 ; p=0.039$ )were independent factors affecting OS in patients with ESCC (Table 2 and Table 3 ).

\section{A New Risk Score Model Based on LDH Level}

A new risk score model incorporating LDH level, tumor length, $\mathrm{CN}$ stage, $\mathrm{cM}$ stage and Cyfra21-1 level were displayed, which were determined as independent prognostic factors in multivariate analysis for OS. Group 1, Group 2, Group 3 were defined as follows: patients with none or one of these risk prognostic factors, patients with two of these risk prognostic factors and patients with three to five of these risk prognostic factors, respectively. According to this predicting model, patients were stratified into three risk groups with obvious prognosis, as shown in Figure 4.

\section{Discussion}

Recent studies have revealed that increased LDH is related to cancer progression in several solid tumors. $^{8-14,25}$ Several studies also have evaluated the prognostic value of LDH in ESCC. ${ }^{15-20}$ Nevertheless, the prognostic role for pretreatment serum LDH in ESCC remains uncertain until now. The current study demonstrated that lower LDH levels were associated with a better prognosis compared with higher LDH levels, as shown in both multivariable analysis based on the whole cohort of 614 patients and the propensity score-matched cohort of 256 patients. More importantly, the study was one of the few 
reports which established a new risk prognostic scoring model based on the baseline LDH level, which stratified patients into three groups with different prognosis. Meanwhile, our study found that elevated LDH was in fact related with more distant metastasis and larger tumor length, suggesting that a high level LDH was more likely to reflect a heavier tumor burden and may represent a more aggressive disease in ESCC.

Based on our current data, pretreatment serum LDH levels appeared to predict the clinical outcome of OS and PFS in the multivariate analysis, which further confirmed the prognostic value of LDH in ESCC patients undergoing chemoradiotherapy. The result was in accordance with two previously published analyses suggesting a relationship between LDH levels and a worse outcome in ESCC. ${ }^{15,18}$ However, this opposited with the results of another retrospective study on 212 patients with ESCC undergoing chemoradiotherapy by Zhang $\mathrm{P}$ et al that revealed that LDH was not associated with OS or PFS. ${ }^{16}$ One possible explanation was that the discordant method to determine the optimal cut-off value of LDH. In the previous two studies mentioned above, the optimal cut-off values were calculated by cut-off finder or the upper limit of normal (ULN), while Zhang P et al did not explain the reason for the cut-off value of $\mathrm{LDH}$ that maybe influence the result to some extent. It also should be noted that there was no standard point for optimal cut-off value of $L D H$, which may be affected by various conditions in clinical practice. Thus, more prospective studies are urgently need to solve the problem of inconsistent optimal LDH cutoff values. Additionally, an investigation that recruited 567 ESCC patients by Luo HS et al has also demonstrated that an elevated LDH level was an independent indicator for poor prognosis. ${ }^{20} \mathrm{To}$ sum up, though no agreement regarding pretreatment serum LDH level was the predictor for prognosis in ESCC, it may be a reliable factor in predicting the therapeutic effect of chemoradiotherapy in patients with ESCC according to our results. More researches are warranted to further validation before widely used in clinical settings.

In addition, according to the result of Cox multivariate analysis, we found that tumor length was another independent prognostic factor for OS and PFS besides LDH, while advanced $\mathrm{cN}$ stage and advanced $\mathrm{cM}$ stage were shown as adverse OS prognostic factors. This concurred with the findings of previous studies by Wei $\mathrm{XL}$ et al. ${ }^{16}$ What made it different was our findings show that $\mathrm{OS}$ is related to tumor length, while theirs were related to tumor differentiation. In a similar study, Yu L et al ${ }^{26}$ supported tumor length, $T$ stage and $\mathrm{M}$ stage were independent prognostic factors for ESCC patients. It was explicable that patient selection in our group only included the ESCC patients underwent chemoradiotherapy, while more than $80 \%$ of the patients underwent curative esophagectomy in their previous retrospective studies. Although the TNM staging and tumor differentiation were identified by EUS, enhanced-scanning CT, PET-CT or pathological biopsy, it was not completely equivalent to postoperative pathological staging.

With regard to Cyfra21-1 in our study, multivariate analysis indicted that a high level of Cyfra21-1 was an adverse prognostic factor and it was better than CEA as a predictor for prognosis in ESCC. Our study emphasized the prognostic value of Cyfra21-1 in predicting OS and PFS. The finding was in accordance with two previously published studies. ${ }^{27,28}$ However, Yang Y reported the contrary conclusion that CEA 
may be superior to other tumor biomarkers as prognostic indicators in ESCC. ${ }^{29}$ More homogeneous studies are needed to confirm the results.

Recently, Luo HS et al proposed a prognostic risk scoring model included the LDH level and neutrophil count to help verify the prognosis for ESCC patients. ${ }^{20}$ However, there was no research about the role of the LDH level combined with tumor markers in the prognosis of ESCC patients treated with chemoradiotherapy. Based on the existing evidence in our study, we established a model based on serum LDH level, tumor biomarkers along with TNM staging system to choose ESCC patients who were most likely to benefit from chemoradiotherapy. Nevertheless, there was no widely used predictive model about prognosis has been constructed in ESCC patients received chemoradiotherapy until now, develop a more effective and reliable prediction model for estimating the prognosis would be our main focus in the subsequent studies.

In conclusion, our study also has some limitations. Firstly, it was a single-center retrospective study and its design tended to have some degree of selection bias. Secondly, it was difficult to obtain complete pathological data for the patients in this study who underwent chemoradiotherapy with non-surgical. Thirdly, the role of LDH in predicting prognosis was limited owing to some other factors influencing the LDH levels except for the tumors. Moreover, the most sensitive cut-off points of serum LDH required largescale clinical trials to define. Therefore, a multicenter, large-sample prospective study is needed to further verify the conclusions before this method can be applied to routine clinical studies. We believe that LDH should be considered as a relevant clinical variable to be included in the prognostic classification of ESCC patients, with the aim to better determine the most appropriate treatment strategies and to better stratify patients included in clinical trials.

\section{Abbreviations}

EC

Esophageal cancer; ESCC:esophageal squamous cell carcinoma; OS:overall survival; PFS:progressionfree survival; LDH:lactate dehydrogenase; PS:performance status; Cyfra21-1:cytokeratin 19 fragment antigen 21 - 1; CEA:carcinoembryonic antigen; 3D-CRT:three-dimensional conformal radiotherapy; IMRT:intensity modulated radiotherapy; CCRT:concurrent chemoradiotherapy; SCRT:sequential chemoradiotherapy (SCRT); cT stage:clinical T stage ; cN stage:clinical N stage; cM stage:clinical M stage; ECOG:Eastern Clinical Oncology Group; NCCN:National Comprehensive Cancer Network;

\section{Declarations}

\section{Ethics approval and consent to participate}

This study was approved by the Research Ethics Board of Shandong Cancer Hospital, and informed consent was provided by all patients. 
All authors gave their consent for publication.

\section{Availability of data and materials}

The datasets used and analyzed during the current study are available from the corresponding author on reasonable request.

\section{Conflict of interest}

The authors declare that they have no competing interests.

\section{Funding}

Not applicable.

\section{Authors' contributions}

JMH and TZ participated in the study design, collected the clinical data, performed the statistical analysis and drafted the manuscript. CXL and BSL conceived the study, participated in its design and revised the manuscript. All authors read and approved the final manuscript.

\section{Acknowledgements}

During the protocol designing, data collecting and analyzing, and the manuscript writing, tremendous supports had been being from clinical and technique colleagues. Their supports and helps should be appreciated.

\section{References}

[1] Ferlay J, Soerjomataram I, Dikshit R, et al.Cancer incidence and mortality worldwide: sources, methods and major patterns in GLOBOCAN 2012. Int J Cancer. 2015;136(5):E359-86. Doi:10.1002/ijc.29210

[2] Zeng $\mathrm{H}$, Chen W, Zheng R, et al. Changing cancer survival in China during 2003-15: a pooled analysis of 17 population-based cancer registries.Lancet Glob Health. 2018;6:e555-e567. Doi:10.1016/s2214$109 x(18) 30127-x$

[3] Kamangar, F., Nasrollahzadeh, D., Safiri, S., (2020). The global, regional, and national burden of oesophageal cancer and its attributable risk factors in 195 countries and territories, 1990-2017: a systematic analysis for the Global Burden of Disease Study 2017. The Lancet Gastroenterology \&Hepatology.

[4] Lin Y, Totsuka Y, He Y, et al. Epidemiology of esophageal cancer in Japan and China. J Epidemiol. 2013; 23: 233-42. Doi:10.2188/jea.je20120162 
[5] Thabault L, Brisson L, Brustenga C, et al.Interrogating the Lactate Dehydrogenase Tetramerization Site Using (Stapled) Peptides.J. Med. Chem. 2020 May 14;63(9).Doi冈10.1021/acs.jmedchem.9b01955

[6] Urbanska K, Orzechowski A. Unappreciated role of LDHA and LDHB to control apoptosis and autophagy in tumor cells. Int J Mol Sci. (2019) 20. Doi:10.3390/ijms20092085

[7] Van Wilpe, S., Koornstra, R., Den Brok, M. , et al. Lactate dehydrogenase: a marker of diminished antitumor immunity. Oncolmmunology, 2020;9(1):1731942. Doi:10.1080/2162402x.2020.1731942

[8] Alkhawtani R. H. M., Noordzij W. , Glaudemans A. W. J. M., et al. Lactate dehydrogenase levels and 18F-FDG PET/CT metrics differentiate between mediastinal Hodgkin's lymphoma and primary mediastinal B-cell lymphoma. Nuclear Medicine Communications, 2018;39(6):572-578.

[9] Chen, Z., Qiu, M., Wu, X., et al. Elevated baseline serum lactate dehydrogenase indicates a poor prognosis in primary duodenum adenocarcinoma patients. J Cancer. 2018; 9: 512-20.

Doi:10.7150/jca.22305

[10] Petrelli, F., Ardito, R., Merelli, B, et al. Prognostic and predictive role of elevated lactate dehydrogenase in patients with melanoma treated with immunotherapy and BRAF inhibitors: a systematic review and meta-analysis.Melanoma Res. 2019;29:1-12. Doi:10.1097/cmr.0000000000000520

[11] Chen B., Dai D., Tang H., et al. Pre-treatment serum alkaline phosphatase and lactate dehydrogenase as prognostic factors in triple negative breast cancer. Journal of Cancer, 2016;7(15): 2309-2316. Doi:10.7150/jca.16622

[12] Long G., Tang W., Fu X., et al. Pre-treatment Serum Lactate Dehydrogenase Predicts Distant Metastasis and Poor Survival in Nasopharyngeal Carcinoma. Journal of Cancer, 2019;10(16): 36573664. Doi:10.7150/jca.32716

[13] Wang C, Jin S, Xu S, Cao S.High Systemic Immune-Inflammation Index (SII) Represents an Unfavorable Prognostic Factor for Small Cell Lung Cancer Treated with Etoposide and Platinum-Based Chemotherapy. Lung 2020 Apr;198(2). Doi:

$10.1007 / \mathrm{s} 00408-020-00333-6$

[14] Ji, F., Fu, S.-J., Guo, Z.-Y., et al. Prognostic value of combined preoperative lactate dehydrogenase and alkaline phosphatase levels in patients with resectable pancreatic ductal adenocarcinoma. Medicine (Baltimore). 2016;95:e4065. Doi:10.1097/MD.0000000000004065

[15] Wei XL, Zhang DS, He MM, et al. The predictive value of alkaline phosphatase and lactate dehydrogenase for overall survival in patients with esophageal squamous cell carcinoma. Tumour Biol. 2016;37:1879-87. Doi: 10.1007/s13277-015-3851-y 
[16] Zhang P., Xi M., Li QQ., et al. The modified Glasgow prognostic score is an independent prognostic factor in patients with inoperable thoracic esophageal squamous cell carcinoma undergoing chemoradiotherapy. J Cancer Educ. 2014;5:689-95.Doi:10.7150/jca.9569

[17] Feng JF , Wang L , Yang X , Jiang YH. Prognostic value of lactate dehydrogenase to albumin ratio (LAR) in patients with resectable esophageal squamous cell carcinoma. Cancer Manag Res. $2019 \mathrm{Jul}$ 31;11:7243-7251. Doi:10.2147/CMAR.S208320

[18] Wang X.,Zhang B., Chen X. et al. Lactate dehydrogenase and baseline markers associated with clinical outcomes of advanced esophageal squamous cell carcinoma patients treated with camrelizumab (SHR-1210), a novel anti-PD-1 antibody.Thorac. Cancer 2019, 10: 1395-1401. Doi:10.1111/1759-

7714.13083

[19] Feng JF , Wang L , Yang X ,Chen S. Gustave Roussy Immune Score (GRIm-Score) is a prognostic marker in patients with resectable esophageal squamous cell carcinoma. J Cancer.2020;11(6):13341340. Doi: $10.7150 /$ jca.37898

[20]Luo HS, Xu HY, Du ZS, et al.Prognostic Significance of Baseline Neutrophil Count and Lactate Dehydrogenase Level in Patients With Esophageal Squamous Cell Cancer Treated With Radiotherapy. Front Oncol 2020;10 Doi冈10.3389/fonc.2020.00430

[21] Rice, T. W., Rusch, V. W., Ishwaran, H., et al. Cancer of the esophagus and esophagogastric junction: data-driven staging for the seventh edition of the American joint committee on cancer/international union against cancer staging manuals. Cancer. 2010;116(16):3763-3773. Doi:10.1002/cncr.25146

[22] Oken, M. M., Creech, R. H., Tormey, D. C., et al. Toxicity and response criteria of the Eastern cooperative oncology group. Am J Clin Oncol.1982;5(6):649-656. Doi:10.1097/00000421-19821200000014

[23] Camp RL, Dolled-Filhart M, Rimm DL. X-tile: a new bio-informatics tool for biomarker assessment and outcome-based cut-point optimization. Clinical cancer research : an official journal of the American Association for Cancer Research. 2004;10(21):7252-9.Doi: 10.1158/1078-0432.CCR-04-0713

[24] D'Agostino RB. Propensity score methods for bias reduction in the comparison of a treatment to a non-randomized control group. Stat Med 1998; 17:2265-2281. Doiه10.1002/(sici)10970258(19981015)17:19<2265::aid-sim918>3.0.co;2-b

[25] Deme D, Telekes A. Prognostic importance of lactate dehydrogenase (LDH) in oncology. Orv Hetil 2017.(In Hungarian.); 158: 1977-88.

[26] Yu L, Zhang XT, Guan SH, Cheng YF, Li LX. The number of negative lymph nodes is positively associated with survival in esophageal squamous cell carcinoma patients in China. Open Med. 2020; 15: 152-159.Doi: 10.1515/med-2020-0023 
[27] Zhang HQ, Wang RB, Yan HJ, Zhao W, Zhu KL, Jiang SM, et al.Prognostic significance of CYFRA21$1, C E A$ and hemoglobin in patients with esophageal squamous cancer undergoing concurrent chemoradiotherapy. Asian Pac J Cancer Prev. 2012;13(1):199-203.

41.38.Doi:10.7314/apjcp.2012.13.1.199

[28] Yan HJ, Wang RB, Zhu KL, Jiang SM, Zhao W, Xu XQ, et al. Cytokeratin 19 fragment antigen 21-1 as an independent predictor for definitive chemoradiotherapy sensitivity in esophageal squamous cell carcinoma. Chin Med J. 2012;125(8):1410-5.

[29] Yang Y, Huang X, Zhou L, Deng T, Ning T, Liu R, et al. Clinical use of tumor biomarkers in prediction for prognosis and chemotherapeutic effect in esophageal squamous cell carcinoma.BMC Cancer 2019 May 31;19(1). Doi:10.1186/s12885-019-5755-5

\section{Tables}

Table 1 Clinical Features of ESCC patients based on LDH Before and After Propensity Score Matching 


\begin{tabular}{|c|c|c|c|c|c|c|}
\hline \multirow[b]{2}{*}{ Variables } & \multicolumn{3}{|c|}{ Before matching } & \multicolumn{3}{|c|}{ After matching } \\
\hline & $\begin{array}{l}\text { Low-LDH } \\
(\mathrm{n}=68)\end{array}$ & $\begin{array}{l}\text { High-LDH } \\
(\mathrm{n}=546)\end{array}$ & $\begin{array}{l}P \\
\text { value }\end{array}$ & $\begin{array}{l}\text { Low-LDH } \\
(\mathrm{n}=68)\end{array}$ & $\begin{array}{l}\text { High-LDH } \\
(\mathrm{n}=188)\end{array}$ & $\begin{array}{l}P \\
\text { value }\end{array}$ \\
\hline \multicolumn{7}{|l|}{ Gender } \\
\hline Male & 57 & 421 & \multirow[t]{2}{*}{0.208} & 57 & 153 & \multirow[t]{2}{*}{0.653} \\
\hline Female & 11 & 125 & & 11 & 35 & \\
\hline \multicolumn{7}{|l|}{ Age (years) } \\
\hline$\leq 69$ & 58 & 427 & \multirow[t]{2}{*}{0.176} & 58 & 162 & \multirow[t]{2}{*}{0.859} \\
\hline$>69$ & 10 & 119 & & 10 & 26 & \\
\hline \multicolumn{7}{|l|}{ ECOG PS } \\
\hline 0 & 34 & 236 & \multirow[t]{2}{*}{0.288} & 34 & 99 & \multirow[t]{2}{*}{0.707} \\
\hline $1-2$ & 34 & 310 & & 34 & 89 & \\
\hline \multicolumn{7}{|l|}{ cT stage } \\
\hline T1-2 & 6 & 53 & \multirow[t]{2}{*}{0.816} & 6 & 18 & \multirow[t]{2}{*}{0.856} \\
\hline T3-4 & 62 & 493 & & 62 & 170 & \\
\hline \multicolumn{7}{|l|}{ cN stage } \\
\hline No & 10 & 67 & \multirow[t]{2}{*}{0.568} & 10 & 23 & \multirow[t]{2}{*}{0.602} \\
\hline $\mathrm{N}+$ & 58 & 479 & & 58 & 165 & \\
\hline \multicolumn{7}{|l|}{ cM stage } \\
\hline MO & 62 & 417 & \multirow[t]{2}{*}{0.005} & 62 & 172 & \multirow[t]{2}{*}{0.937} \\
\hline M1 & 6 & 129 & & 6 & 16 & \\
\hline \multicolumn{7}{|l|}{ Differentiation } \\
\hline High & 61 & 442 & \multirow[t]{2}{*}{0.077} & 61 & 172 & \multirow[t]{2}{*}{0.659} \\
\hline Moderate or Poor & 7 & 104 & & 7 & 16 & \\
\hline \multicolumn{7}{|l|}{ Length (cm) } \\
\hline$\leq 6.5$ & 48 & 447 & \multirow[t]{2}{*}{0.026} & 48 & 138 & \multirow[t]{2}{*}{0.655} \\
\hline$₫ 6.5$ & 20 & 99 & & 20 & 50 & \\
\hline \multicolumn{7}{|l|}{ Tumor Location } \\
\hline Cervical & 6 & 59 & \multirow[t]{2}{*}{0.308} & 6 & 23 & \multirow[t]{2}{*}{0.523} \\
\hline Upper & 24 & 156 & & 24 & 59 & \\
\hline
\end{tabular}




\begin{tabular}{|c|c|c|c|c|c|c|}
\hline Medium & 22 & 232 & & 22 & 73 & \\
\hline Lower & 16 & 99 & & 16 & 33 & \\
\hline \multicolumn{7}{|c|}{$\begin{array}{l}\text { Radiotherapy } \\
\text { technology }\end{array}$} \\
\hline 3DCRT & 21 & 204 & \multirow[t]{2}{*}{0.296} & 21 & 64 & \multirow[t]{2}{*}{0.635} \\
\hline IMRT & 47 & 342 & & 47 & 124 & \\
\hline \multicolumn{7}{|c|}{ Treatment options } \\
\hline CCRT & 24 & 255 & & 24 & 77 & \\
\hline SCRT & 44 & 291 & 0.075 & 44 & 111 & 0.413 \\
\hline \multicolumn{7}{|c|}{ Total dose (Gy) } \\
\hline$\leq 58.8$ & 12 & 138 & \multirow[t]{2}{*}{0.167} & 12 & 29 & \multirow[t]{2}{*}{0.669} \\
\hline$>58.8$ & 56 & 408 & & 56 & 159 & \\
\hline \multicolumn{7}{|c|}{ CEA (ng/ml) } \\
\hline$\leq 2.4$ & 25 & 190 & \multirow[t]{3}{*}{0.651} & 25 & 62 & \multirow[t]{3}{*}{0.552} \\
\hline$>2.4$ & 21 & 199 & & 21 & 72 & \\
\hline NA & 22 & 157 & & 22 & 54 & \\
\hline \multicolumn{7}{|c|}{ Cyfra21-1 (ng/ml) } \\
\hline$\leq 6.4$ & 35 & 311 & \multirow[t]{3}{*}{0.150} & 35 & 103 & \multirow[t]{3}{*}{0.665} \\
\hline$>6.4$ & 2 & 41 & & 2 & 9 & \\
\hline NA & 31 & 194 & & 31 & 76 & \\
\hline
\end{tabular}

LDH: lactate dehydrogenase; ECOG: Eastern Clinical Oncology Group; PS: performance status; Cyfra21-1: cytokeratin 19 fragment antigen 21-1; CEA : carcinoembryonic antigen; 3D-CRT: three-dimensional conformal radiotherapy; IMRT: intensity modulated radiotherapy; CCRT: concurrent chemoradiotherapy;SCRT: sequential chemoradiotherapy (SCRT); CT stage: clinical T stage ; $\mathrm{CN}$ stage: clinical $\mathrm{N}$ stage ; cM stage: clinical M stage;

Table 2 Univariate and multivariate analysis of PFS in patients with ESCC $(\mathrm{N}=256)$ after PSM 


\begin{tabular}{|c|c|c|c|c|}
\hline \multirow{2}{*}{$\begin{array}{l}\text { Progression-free survival } \\
\text { Variable }\end{array}$} & \multicolumn{2}{|l|}{ Univariate } & \multicolumn{2}{|l|}{ Multivariate } \\
\hline & $\mathrm{HR}(95 \% \mathrm{Cl})$ & $\begin{array}{l}P \\
\text { value }\end{array}$ & $\mathrm{HR}(95 \% \mathrm{Cl})$ & $P$ value \\
\hline Gender (Female vs Male) & $\begin{array}{l}0.99 \\
(0.66,1.49)\end{array}$ & 0.960 & - & - \\
\hline Age (>69 vs $\leq 69$, year) & $\begin{array}{l}1.16 \\
(0.75,1.81)\end{array}$ & 0.501 & - & - \\
\hline ECOG PS (1-2 vs 0) & $\begin{array}{l}1.17 \\
(0.86,1.61)\end{array}$ & 0.324 & - & - \\
\hline cT stage (T3-4 vs T1-2) & $\begin{array}{l}1.06 \\
(0.61,1.83)\end{array}$ & 0.843 & - & - \\
\hline cN stage (N+ vs N0) & $\begin{array}{l}1.54 \\
(0.92,2.58)\end{array}$ & 0.104 & - & - \\
\hline cM stage (M1 vs M0) & $\begin{array}{l}1.40 \\
(0.82,2.39)\end{array}$ & 0.213 & - & - \\
\hline Differentiation (Moderate/poor vs high) & $\begin{array}{l}1.40 \\
(0.83,2.35)\end{array}$ & 0.204 & - & - \\
\hline Tumor length $(16.5 \mathrm{vs} \leq 6.5, \mathrm{~cm})$ & $\begin{array}{l}1.58 \\
(1.12,2.21)\end{array}$ & 0.009 & $1.62(1.15,2.28)$ & 0.005 \\
\hline Tumor Location & & 0.108 & & \\
\hline upper vs Cervical & $\begin{array}{l}0.60 \\
(0.36,1.03)\end{array}$ & 0.062 & - & - \\
\hline Medium vs Cervical & $\begin{array}{l}0.82 \\
(0.49,1.36)\end{array}$ & 0.437 & - & - \\
\hline Lower vs Cervical & $\begin{array}{l}1.00 \\
(0.58,1.74)\end{array}$ & 0.991 & - & - \\
\hline $\begin{array}{l}\text { Radiotherapy technology (IMRT vs } \\
\text { 3DCRT) }\end{array}$ & $\begin{array}{l}0.83 \\
(0.60,1.15)\end{array}$ & 0.263 & - & - \\
\hline Treatment options (CCRT vs SCRT) & $\begin{array}{l}1.29 \\
(0.93,1.79)\end{array}$ & 0.126 & & \\
\hline Dose (58.8> vs $\leq 58.8$, Gy) & $\begin{array}{l}0.78 \\
(0.52,1.17)\end{array}$ & 0.234 & - & - \\
\hline \multicolumn{5}{|l|}{$\mathrm{CEA}(\mathrm{ng} / \mathrm{ml})$} \\
\hline$>5.5$ vs $\leq 5.5$ & $\begin{array}{l}1.09 \\
(0.74,1.59)\end{array}$ & 0.668 & - & - \\
\hline NA vs $\leq 5.5$ & $\begin{array}{l}1.04 \\
(0.70,1.54)\end{array}$ & 0.855 & - & - \\
\hline Cyfra21-1 (ng/ml) & & & & \\
\hline
\end{tabular}




\begin{tabular}{|c|c|c|c|c|}
\hline$>6.4$ vs $\leq 6.4$ & $\begin{array}{l}2.49 \\
(1.29,4.83)\end{array}$ & 0.007 & $\begin{array}{l}2.37(1.22 \\
, 4.60)\end{array}$ & 0.011 \\
\hline NA vs $\leq 6.4$ & $\begin{array}{l}0.80 \\
(0.58,1.12)\end{array}$ & 0.194 & $0.80(0.58,1.12)$ & 0.196 \\
\hline LDH $(>134$ vs $\leq 134, \mathrm{U} / \mathrm{L})$ & $\begin{array}{l}1.51 \\
(1.03,2.20)\end{array}$ & 0.033 & $1.50(1.03,2.19)$ & 0.035 \\
\hline
\end{tabular}

HR, hazard ratio; Cl: confidence interval; LDH: lactate dehydrogenase; ECOG: Eastern Clinical Oncology Group; PS: performance status; Cyfra21-1: cytokeratin 19 fragment antigen 21-1; CEA : carcinoembryonic antigen; 3D-CRT: three-dimensional conformal radiotherapy; IMRT: intensity modulated radiotherapy; CCRT: concurrent chemoradiotherapy; SCRT: sequential chemoradiotherapy (SCRT);CT stage: clinical T stage ; $c N$ stage: clinical $\mathrm{N}$ stage ; $\mathrm{cM}$ stage: clinical M stage; ESCC: esophageal squamous cell carcinoma; PFS: progression-free survival;

Table 3 Univariate and multivariate analysis of OS in patients with ESCC $(\mathrm{N}=256)$ after PSM 


\begin{tabular}{|c|c|c|c|c|}
\hline \multirow{2}{*}{$\begin{array}{l}\text { Overall survival } \\
\text { Variable }\end{array}$} & \multicolumn{2}{|l|}{ Univariate } & \multicolumn{2}{|l|}{ Multivariate } \\
\hline & $\mathrm{HR}(95 \% \mathrm{Cl})$ & $P$ value & $\mathrm{HR}(95 \% \mathrm{Cl})$ & $P$ value \\
\hline Gender (Female vs Male) & $0.89(0.56,1.41)$ & 0.625 & - & - \\
\hline Age (>69 vs $\leq 69$, year) & $1.04(0.63,1.70)$ & 0.891 & - & - \\
\hline ECOG PS (1-2 vs 0) & $1.17(0.83,1.65)$ & 0.368 & - & - \\
\hline cT stage (T3-4 vs T1-2) & $0.87(0.49,1.54)$ & 0.628 & - & - \\
\hline cN stage (N+ vs N0) & $2.29(1.20,4.38)$ & 0.012 & $1.94(1.01,3.72)$ & 0.047 \\
\hline cM stage (M1 vs M0) & $1.88(1.10,3.22)$ & 0.022 & $2.04(1.18,3.53)$ & 0.011 \\
\hline Differentiation (Moderate/poor vs high) & $1.40(0.80,2.44)$ & 0.236 & - & - \\
\hline Tumor length $(06.5 \mathrm{vs} \leq 6.5, \mathrm{~cm})$ & $1.56(1.08,2.26)$ & 0.017 & $1.61(1.11,2.34)$ & 0.013 \\
\hline \multicolumn{5}{|l|}{ Tumor Location } \\
\hline upper vs Cervical & $0.68(0.39,1.20)$ & 0.134 & - & - \\
\hline Medium vs Cervical & $0.68(0.39,1.20)$ & 0.134 & - & - \\
\hline Lower vs Cervical & $1.04(0.57,1.88)$ & 0.904 & - & - \\
\hline $\begin{array}{l}\text { Radiotherapy technology (IMRT vs } \\
\text { 3DCRT) }\end{array}$ & $0.80(0.56,1.14)$ & 0.220 & - & - \\
\hline Treatment options (CCRT vs SCRT) & $\begin{array}{l}1.43 \\
(0.99,1.79)\end{array}$ & 0.054 & - & - \\
\hline Dose (58.8> vs $\leq 58.8$, Gy) & $0.75(0.48,1.17)$ & 0.206 & - & - \\
\hline \multicolumn{5}{|l|}{$\operatorname{CEA}(\mathrm{ng} / \mathrm{ml})$} \\
\hline$>5.5$ vs $\leq 5.5$ & $1.08(0.71,1.63)$ & 0.733 & - & - \\
\hline NA vs $\leq 5.5$ & $1.13(0.74,1.73)$ & 0.584 & - & - \\
\hline \multicolumn{5}{|l|}{ Cyfra21-1 (ng/ml) } \\
\hline$>6.4$ vs $\leq 6.4$ & $3.17(1.63,6.18)$ & 0.001 & $2.81(1.43,5.50)$ & 0.003 \\
\hline NA vs $\leq 6.4$ & $0.98(0.68,1.40)$ & 0.895 & $1.00(0.70,1.44)$ & 0.983 \\
\hline LDH (>134 vs $\leq 134, \mathrm{U} / \mathrm{L})$ & $1.55(1.02,2.35)$ & 0.040 & $1.56(1.02,2.39)$ & 0.039 \\
\hline
\end{tabular}

HR, hazard ratio; Cl: confidence interval; LDH: lactate dehydrogenase; ECOG: Eastern Clinical Oncology Group; PS: performance status; Cyfra21-1: cytokeratin 19 fragment antigen 21-1; CEA : carcinoembryonic antigen; 3D-CRT: three-dimensional conformal radiotherapy; IMRT: intensity modulated radiotherapy; CCRT: concurrent chemoradiotherapy;SCRT: sequential chemoradiotherapy (SCRT);CT stage: clinical T 
stage ; $c N$ stage: clinical $\mathrm{N}$ stage ; $\mathrm{cM}$ stage: clinical M stage; ESCC: esophageal squamous cell carcinoma; PFS: progression-free survival;

\section{Figures}
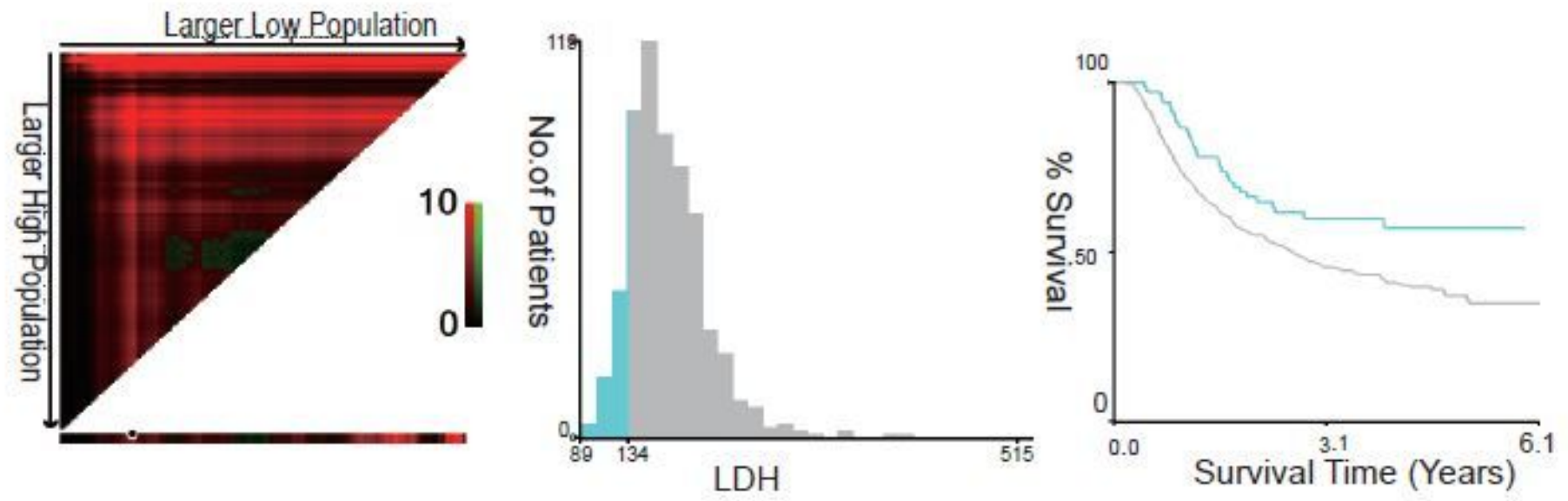

Figure 1

X-tile analyses. The optimum cut-off points for LDH was $134 \mathrm{U} / \mathrm{L}$ according to the X-tile program. Abbreviations: LDH, lactate dehydrogenase;
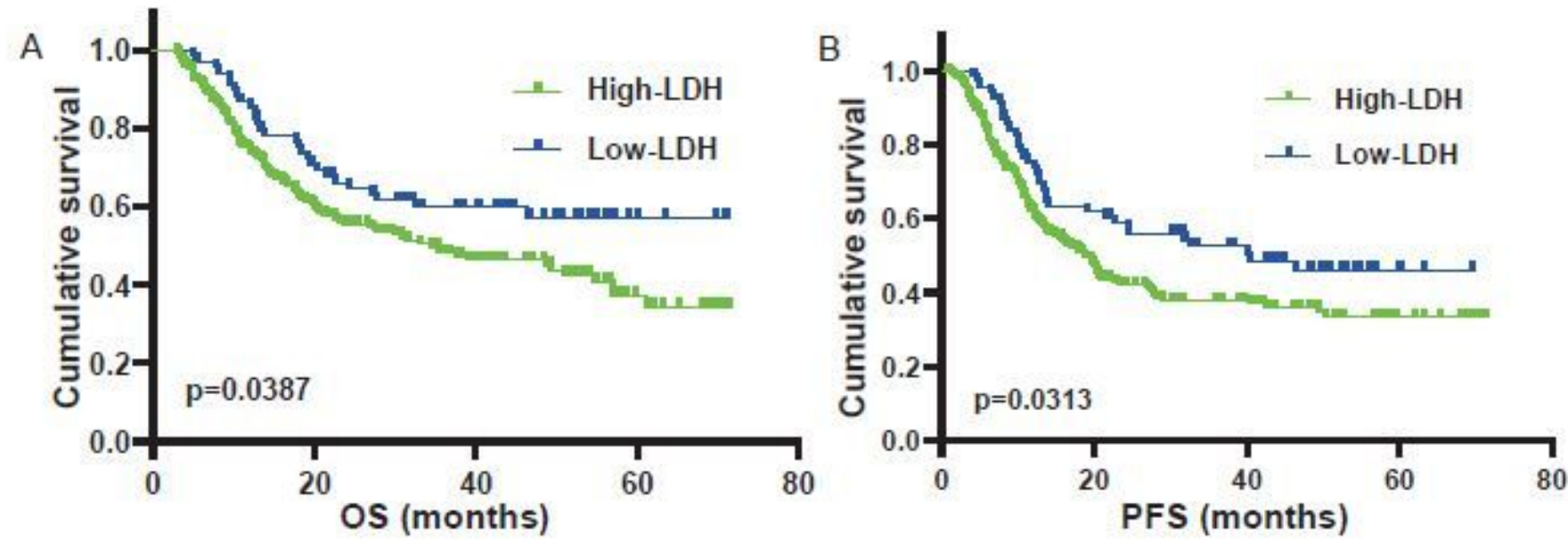

\section{Figure 2}

Kaplan-Meier survival curves of OS and PFS grouped by LDH for 256 patients in the matched cohort. (A)The OS curve of ESCC patients underwent chemoradiotherapy classified by LDH; (B) The PFS curve of 
ESCC patients underwent chemoradiotherapy classified by LDH; Abbreviations: OS, overall survival; PFS, progression-free survival; LDH, lactate dehydrogenase;

A

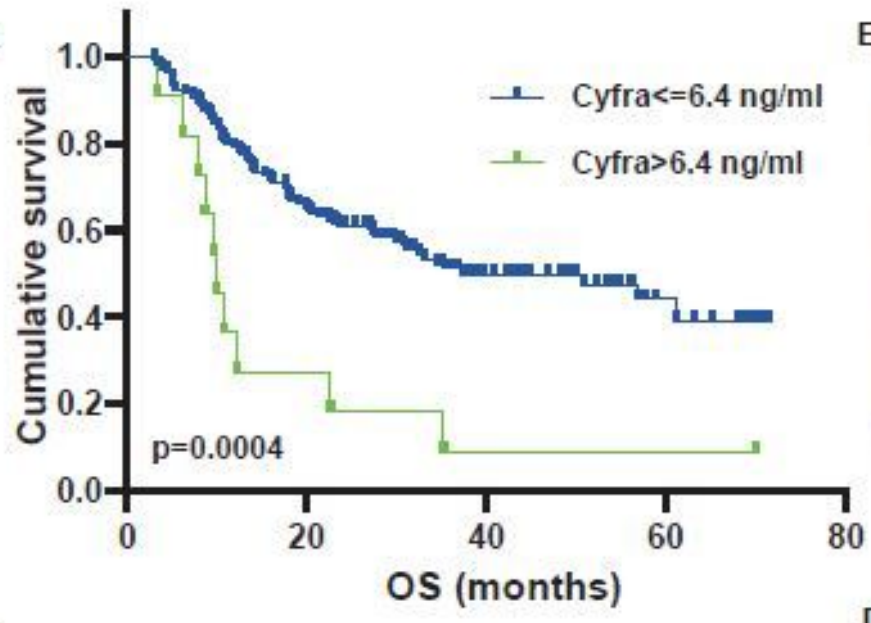

C

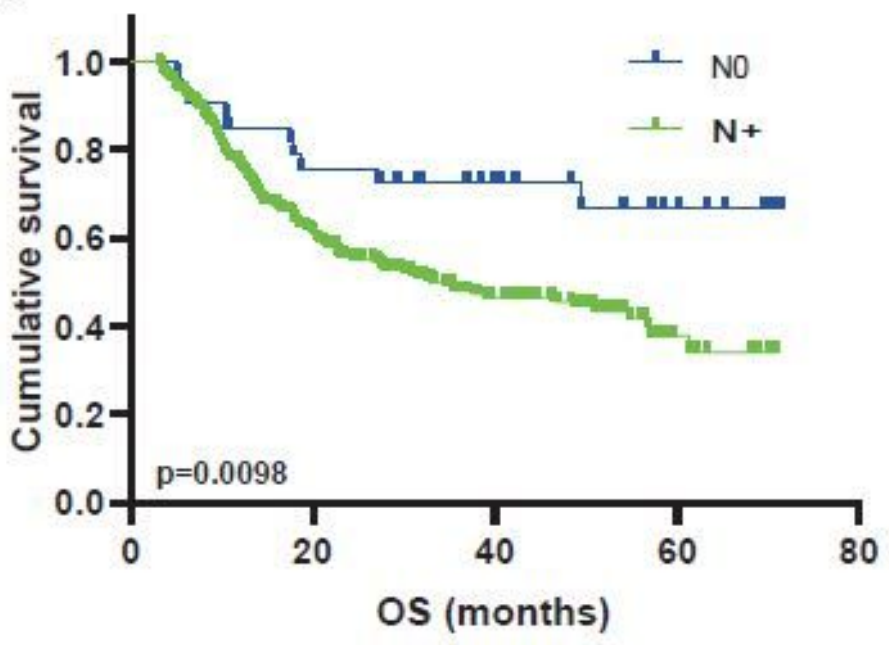

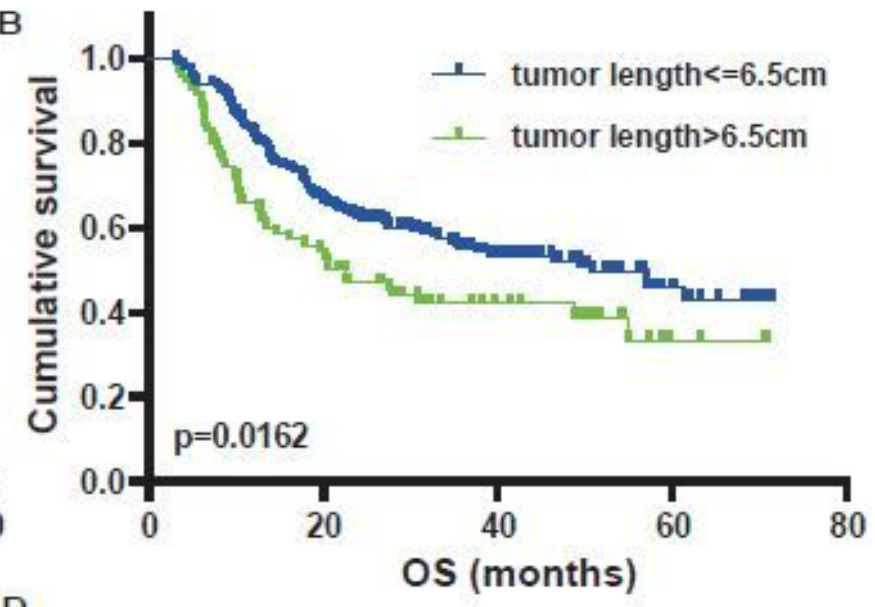

D

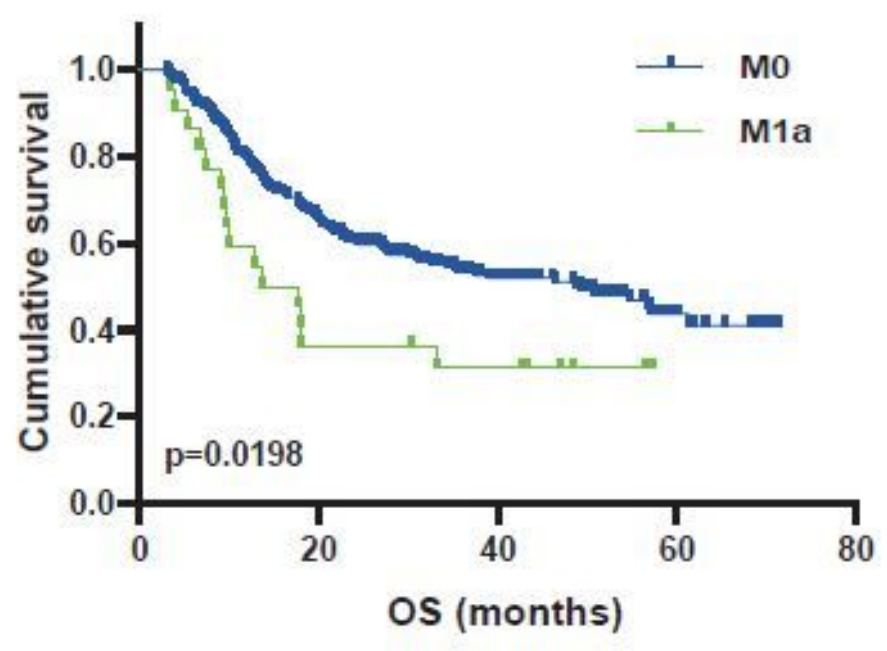

\section{Figure 3}

Kaplan-Meier survival curves of OS for ESCC patients underwent chemoradiotherapy classified according to different prognostic factors. (A) Patients were classified by Cyfra21-1 (Cyfra21-1 $<=6.4 \mathrm{ng} / \mathrm{ml} v \mathrm{~s}$ Cyfra21-1 $>6.4 \mathrm{ng} / \mathrm{ml}$ ). (B) Patients were classified by tumor length (tumor length $<=6.5 \mathrm{~cm}$ vs tumor length $>6.5 \mathrm{~cm}$ ). (C) Patients were classified by $\mathrm{cN}$ stage (NO vs $\mathrm{N}+$ stage). (D) Patients were classified by cM stage (M0 vs M1a stage). 


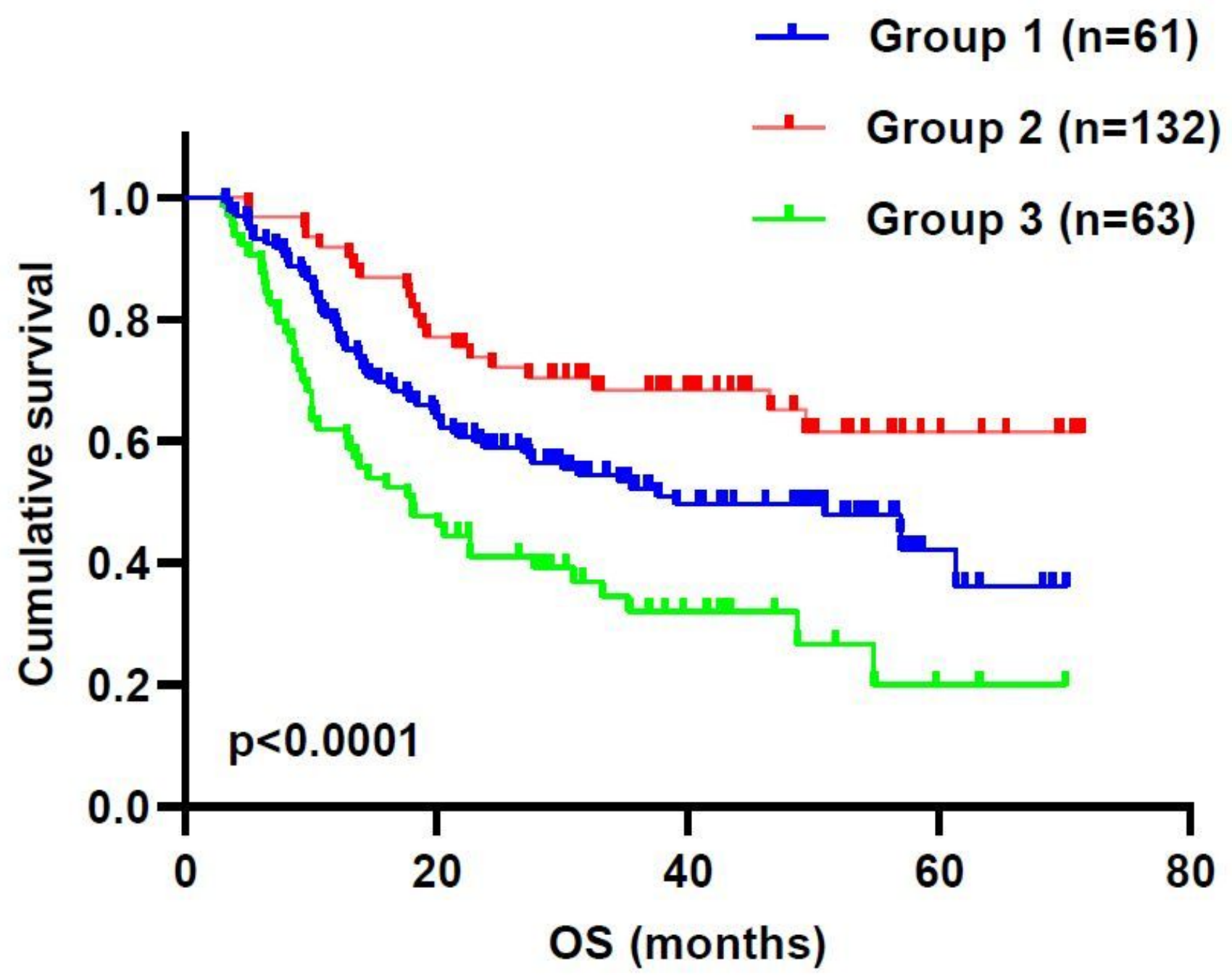

Figure 4

Kaplan-Maier survival curves of overall survival for ESCC patients underwent chemoradiotherapy stratified according to a new risk score model.

\section{Supplementary Files}

This is a list of supplementary files associated with this preprint. Click to download.

- FigureS1.pdf

- SupplementaryMaterial.docx 